\title{
Synthesis and Biological Activity Evaluation of Some New Heterocyclic Spirocompounds with Imidazolinone and Pyrazoline Moieties
}

\author{
Omar A. Miqdad (Corresponding author) \& Nada M. Abunada \\ Department of Chemistry, Faculty of Applied Sciences, Al-Aqsa University \\ 76888, Gaza, Palestine \\ E-mail: miqdadomar@hotmail.com; nadanadannrs@yahoo.com \\ Hamdi M. Hassaneen \\ Department of Chemistry, Faculty of Science, Cairo University, Egypt \\ Ahmed S. M. Abu Samaha \\ Department of Biology, Faculty of Applied Sciences, Al-Aqsa University \\ 76888, Gaza, Palestine
}

Received: August 19, 2011

Accepted: September 8, 2011

Published: December 1, 2011

doi:10.5539/ijc.v3n4p20

URL: http://dx.doi.org/10.5539/ijc.v3n4p20

\begin{abstract}
The synthesis of antimicrobial activity spirocompounds was achieved via the reaction of hydrazonoyl halides $\mathbf{1}$ with exocyclic 4-arylidene-2-methylimidazolin-5-one 3 in benzene in the presence of triethylamine. Correct elemental analyses and spectral data (IR, ${ }^{1} \mathrm{H}$ NMR, ${ }^{13} \mathrm{C}$ NMR and MS) confirm the structure of the synthesized spirocompounds. All the synthesized compounds were evaluated in vitro for their antimicrobial activity against five gram-positive and two gram-negative bacteria using well diffusion method in Mueller-Hinton agar. Most of them exhibited significant antibacterial activity compared with selected standard drugs.
\end{abstract}

Keywords: Spirocompound, Hydrazonoyl halide, Cycloaddition reaction

\section{Introduction}

Spirocompounds form a group of generally less investigated compounds. However, recently growing efforts have been made to synthesize, characterize and investigate the biological activities of these compounds. Many spirocompounds possess very promising biological activities as anticancer agents (Young-Won et al., 2003; Wen-Liang et al., 2007), antibacterial agents (van-der-Sar et al., 2006; Hyeong-Beom et al., 2007), anticonvulsant agents (Jolanta \& Krzysztof, 2006; Krzysztof et al., 2008; Jolanta et al., 2006), anti tuberculosis agents (Chande et al., 2005), anti-Alzheimer's agents (Masakazu et al., 2001), pain-relief agents (Frank et al., 2003; Hans et al., 2006), anti-dermatitis agents (Nakao et al., 2008) and antimicrobial agents (Pawar et al., 2009; Thadhaney et al., 2010). In addition to their medical uses, some spirocompounds have found other uses in the agricultural and industrial fields. For example, they are used as antifungal agents (Hejiao et al., 2006), pesticides (Lindell et al., 2001), laser dyes (Kreuder et al., 1999) and electroluminescent devices (Lupo et al., 2008). Spiro compounds have also been recently used as antioxidants (Sarma et al., 2010; Shimakawa et al., 2003). Also, the pyrazoline derivatives are very interesting compounds due to their important role for the antifungal (Korgaokar et al., 1996; Abunada et al., 2009), antidepressant (Palaska et al., 2003; Rajendra et al., 2005; Ozdemir et al., 2007; Ruhogluo et al., 2005), anticonvulsant (Ozdemir et al., 2007; Ruhogluo et al., 2005), anti-inflammatory (Karabasanagouda et al., 2009), antibacterial (Abunada et al., 2009; Nauduri \& Reddy, 1998) and anti-tumor (Taylor \& Patel, 1992) activity. Besides, it is known that the imidazolinone derivatives are associated with a wide range of therapeutic activities such as anticonvulsant (Godefroi \& Platje, 1972), potent CNS depressant, (Harfenist et al., 1978). Recently some new imidazolinone derivatives have been reported as antimicrobial (Desai et al., 2009; Solankee et al., 2004), anticancer (Solankee et al., 2004) and L-DOPA prodrugs in the treatment of Parkinson's disease (Giorgionia et al., 2010). In view of these results and as continuation of our 
recent work on the synthesis of unreported hitherto spirocompouns (Miqdad et al., 2011), it was therefore considered worthwhile to synthesize some new spirocompound derivatives with pyrazoline residue attach to imidazolinone one in order to find new biologically active molecules. Thus, the synthesis of new tetrazaspiro[4,4]nona-2,8-dien-6-one derivatives has been achieved.

\section{Results and discussion}

Tetrazaspiro[4,4]nona-2,8-dien-6-one derivatives 4a-w were produced via the cycloaddition reaction of nitrilimines $\mathbf{2}$, generated in situ by the action of triethylamine on hydrazonoyl halides 1a-f in dry benzene at reflux temperature with exocyclic double bond of 4-arylidene-1-aryl-2-methyl-1H-imidazol-5(4H)-one 3a-f (Scheme 1). The structures of compounds $\mathbf{4 a - w}$ were established based on the basis of their elemental analyses and spectral data. Their IR spectra showed absorption bands in the region $1705.0-1759.0$ and $1627.8-1651.0$ $\mathrm{cm}^{-1}$ assignable to the stretching bands of $\mathrm{C}=\mathrm{O}$ and $\mathrm{C}=\mathrm{N}$ bonds. Their ${ }^{1} \mathrm{H}$ NMR spectra revealed, besides aromatic protons at $6.88-8.29 \mathrm{ppm}$, imidazolinone methyl at $1.52-1.72 \mathrm{ppm}$ and the pyrazoline ring proton resonates at $5.30-5.63 \mathrm{ppm}$. Comparison of this chemical shift of the 4-H-pyrazoline ring residue proton of 4 with those of the related compounds we recently prepared (Miqdad et al., 2011) showed similarity between their chemical shifts. Since, in the case of cycloaddition took place in the opposite direction to give $\mathbf{5}$, the expected chemical shift of 5-CH proton would shift downfield due to the deshielding effect of the more electronegative nitrogen atom attached directly to the carbon atom bearing to the hydrogen one. The chemical shift of 5-CH proton of pyrazoline has appeared at $5.9-6.0 \mathrm{ppm}$ (Hassaneen et al., 1995; Shawali et al., 1992). The ${ }^{13} \mathrm{C}$ NMR spectra of some selected compounds showed all the signals corresponding to the proposed structures, especially 4- $\mathrm{CH}$ and $\mathrm{C}-5$ (spiro carbons) were found to resonate at $62.42-63.75$ and $89.55-91.13 \mathrm{ppm}$ respectively, and the carbonyl carbon were found to resonate at $178.43-179.57 \mathrm{ppm}$. Also, the electron impact (EI) mass spectra displayed the correct molecular ions in accordance with the suggested structures. It is worthy to mention that all attempts to isolate products from the reaction of $\mathbf{3}$ with hydrazonoyl halides bearing carbonyl group $(\mathrm{RCOC}(\mathrm{X})=\mathrm{NNHAr})$ at the carbon atom failed.

\section{In vitro antimicrobial activity evaluation}

An evaluation of the antibacterial activity using five Gram-negative (Escherichia coli, Pseudomonas aeruginosa, Proteus Species, Salmonella Species, Klebsiella Species) and two Gram-positive bacteria (Viridans Streptococci and Coagulase negative Staphylococcus) which were isolated from clinical samples and was assessed for the twenty three newly synthesized compounds by well diffusion method (Atta-ur-Rahman et al., 2001). Based upon the antimicrobial evaluation data of the synthesized compounds it can be seen that compounds $\mathbf{4 m}, \mathbf{4 q}, \mathbf{4 r}, \mathbf{4 s}$ and $\mathbf{4 u}$ showed significant activity against all the tested microorganisms around $16-25 \mathrm{~mm}$. Compounds $\mathbf{4 r}$ and $\mathbf{4} \mathbf{u}$ are the most effective the compounds in the entire series, although they are not effective against C.s. The highest antibacterial activity of $25 \mathrm{~mm}$ and $24 \mathrm{~mm}$ against E.c. was observed for compounds $\mathbf{4 r}$ and $\mathbf{4 u}$ respectively. Also, compounds $\mathbf{4 i}, \mathbf{4 j}, \mathbf{4 r}$ and $\mathbf{4 u}$ possess maximum activity of 23, 24, 20 and $22 \mathrm{~mm}$ respectively, against V.s. $\mathbf{4 m}$ and $\mathbf{4 s}$ showed highest inhibitory activity of 24 and $22 \mathrm{~mm}$ respectively against C.s. and least activity observed against V.s. Compounds $\mathbf{4 m}, \mathbf{4 v}$ and $\mathbf{4 j}, \mathbf{4 l}$ and $\mathbf{4 r}$ showed highest activity of 24 and $22 \mathrm{~mm}$ against P.s. Also, compounds $\mathbf{4 k}, \mathbf{4 r}$ and $\mathbf{4 n}$ possess the highest activity of $24-20 \mathrm{~mm}$ against S.s. Finally, $\mathbf{4 c}, \mathbf{4 r}$ and $\mathbf{4 u}$ exhibited highest inhibitory activity against K.s. of $23-22 \mathrm{~mm}$. Although, compound $\mathbf{4} \mathbf{c}$ was inactive against P.a., V.s., C.s. and P.s. and possess low activity against E.c. and moderate activity against S.s., it exhibit the highest inhibitory activity of $23 \mathrm{~mm}$ against K.s. The antibacterial activities of most of the tested compounds were found higher than the activity of most selected reference antibiotics.

\section{Experimental}

Melting points were measured on Stuart Scientific electrothermal melting point apparatus SMP1 and are uncorrected. The infrared spectra were recorded in potassium bromide disks on a Pye Unicam SP 3-300 and Shimadzu FT-IR 8101 PC infrared spectrophotometer. The ${ }^{1} \mathrm{H}$ NMR $(300.07 \mathrm{MHz})$ and ${ }^{13} \mathrm{C}$ NMR $(75.45 \mathrm{MHz})$ spectra were recorded in DMSO- $\mathrm{d}_{6}$ on a Mercury-300BB NMR using TMS as the internal reference. Mass spectra were measured on a GCMS-QP $1000 \mathrm{EX}$ spectrometer at $70 \mathrm{eV}$. Elemental analyses were carried out at the Microanalytical Centre of Cairo University, Giza, Egypt. Hydrazonoyl halides 1a, 1b (Wolkoff, 1975), 1c (Shawali \& Hassaneen, 1972), 1d (Hegarty \& Scott, 1966), 1e (Aylward \& Scott, 1969), and 1f (Shawali et al., 1990), and the imidazolinone 3 (Islam et al., 1973; Bhattacharjya et al., 2005) were prepared according to the methods reported in the literature.

\subsection{Reaction of $3 a-f$ with Hydrazonoyl Halides 1 a-f (General Procedure)}

Triethylamine $(0.7 \mathrm{ml}, 5 \mathrm{mmol})$ was added to a solution of the appropriate imidazolinone 3a-f $(5 \mathrm{mmol})$ and hydrazonoyl halides 1a-f $(5 \mathrm{mmol})$ in dry benzene $(50 \mathrm{ml})$ and the resulting mixture was refluxed for $6 \mathrm{~h}$. The 
reaction mixture was filtered while hot to remove triethylamine hydrochloride salt and evaporated under reduced pressure till dryness and the remaining residue was triturated with ethanol $(10 \mathrm{ml})$. The separated solid compound was filtered off and crystallized from a given solvent.

8-Methyl-1,3,4,7-tetraphenyl-1,2,7,9-tetrazaspiro[4, 4]nona-2,8-dien-6-one 4a. White crystals, yield 94\%; mp 220 - $222{ }^{\circ} \mathrm{C}$ (benzene); IR (KBr) $v_{\max } / \mathrm{cm}^{-1} 3039.6$ (aromatic $\mathrm{CH}$ ), 2931.6, 2866.0 (aliphatic $\left.\mathrm{CH}\right), 1751.2(\mathrm{C}=\mathrm{O})$, $1643.2(\mathrm{C}=\mathrm{N}), 1596.9(\mathrm{C}=\mathrm{C}) ;{ }^{1} \mathrm{H}$ NMR $\left(\mathrm{DMSO}_{-} \mathrm{d}_{6}\right) \delta 1.65\left(\mathrm{~s}, 3 \mathrm{H}, \mathrm{CH}_{3}\right.$-imidazolinone), $5.62(\mathrm{~s}, 1 \mathrm{H}$, 4H-pyrazole), 7.16-8.26 (m, 20H, ArH's); MS m/z (\%): 428 (M+28, 24), 336 (42), 323 (30), 249 (21), 213 (23), 172 (18), 118 (70), 91 (39), 77 (100); Anal. Calcd. for $\mathrm{C}_{30} \mathrm{H}_{24} \mathrm{~N}_{4} \mathrm{O}$ : C, 78.92; H, 5.30; N, 12.27. Found: C, 78.63 ; $\mathrm{H}, 5.25 ; \mathrm{N}, 12.04 \%$.

3-(4-Chlorophenyl)-8-methyl-1,4,7-triphenyl-1,2,7,9-tetrazaspiro[4,4]nona-2,8-dien-6-one $\quad$ 4b. Pale yellow crystals, yield 93\%; mp $202-204{ }^{\circ} \mathrm{C}$ (benzene/ethanol); IR (KBr) $v_{\max } / \mathrm{cm}^{-1} 3039.6$ (aromatic $\mathrm{CH}$ ), 2931.6, 2866.0 (aliphatic $\mathrm{CH}), 1751.2(\mathrm{C}=\mathrm{O}), 1643.2(\mathrm{C}=\mathrm{N}), 1596.9(\mathrm{C}=\mathrm{C}) ;{ }^{1} \mathrm{H}$ NMR (DMSO-d $\left.\mathrm{d}_{6}\right) \delta 1.66(\mathrm{~s}, 3 \mathrm{H}$, $\mathrm{CH}_{3}$-imidazolinone), 5.42 (s, 1H, 4H-pyrazole), 7.08-7.59 (m, 19H, ArH's); ${ }^{13} \mathrm{C}$ NMR (DMSO-d $\left.\mathrm{d}_{6}\right) \delta 15.368$ ( $\mathrm{CH}_{3}$-imidazolinone), 62.717 (CH-pyrazole), 91.133 (C-spiro), 115.200, 121.521, 127.125, 127.873, 128.021, 128.285, 128.487, 128.781, 129.143, 129.196, 129.727 (11C, CHArC's), 129.902, 132.553, 132.576, 133.518, 143.376, 148.713, 161.255 (7C, C=N, ArC's), 179.030 (CO); MS m/z (\%): 490 (M+, 23), 492 (8.5), 372 (12), 118 (97), 91 (48), 77 (100); Anal. Calcd. for $\mathrm{C}_{30} \mathrm{H}_{23} \mathrm{ClN}_{4} \mathrm{O}$ : C, 73.39; H, 4.72; Cl, 7.22; N, 11.41. Found: C, 73.40; H, 4.67; N, $11.45 \%$.

3-(2,4-Dichlorophenyl)-8-methyl-1-(4-nitrophenyl)-4,7-diphenyl-1,2,7,9-tetrazaspiro[4,4]nona-2,8-dien-6-one 4c Yellow crystals, yield 55\%; mp $272-274{ }^{\circ} \mathrm{C}$ (dimethylformamide/methanol); IR (KBr) $v_{\max } / \mathrm{cm}^{-1} 3035.7$ (aromatic CH), 2923.9, 2839.0 (aliphatic $\mathrm{CH}), 1751.2(\mathrm{C}=\mathrm{O}), 1651.0(\mathrm{C}=\mathrm{N}), 1593.1(\mathrm{C}=\mathrm{C}) ;{ }^{1} \mathrm{H}$ NMR $\left(\mathrm{DMSO}-\mathrm{d}_{6}\right)$ $\delta 1.66$ (s, 3H, $\mathrm{CH}_{3}$-imidazolinone), 5.39 (s, 1H, 4H-pyrazole), 6.93-7.94 (m, 17H, ArH's); $\mathrm{MS} \mathrm{m} / \mathrm{z}(\%): 570$ (M $\mathrm{M}^{+}$, 9.5), 572 (3), 574 (0.8), 541 (21), 540 (19), 449 (20), 448 (16), 118 (100), 90 (23), 77 (92); Anal. Calcd. for $\mathrm{C}_{30} \mathrm{H}_{21} \mathrm{Cl}_{2} \mathrm{~N}_{5} \mathrm{O}_{3}: \mathrm{C}, 63.17 ; \mathrm{H}, 3.71 ; \mathrm{Cl}, 12.43 ; \mathrm{N}, 12.28$. Found: C, 63.38; H, 3.67; N, $12.30 \%$.

3-(2-Furyl)-8-methyl-1-(4-nitrophenyl)-4,7-diphenyl-1,2,7,9-tetrazaspiro[4,4]nona-2,8-dien-6-one 4d. Yellow crystals, yield 56\%; mp $276-278{ }^{\circ} \mathrm{C}$ (dioxane); IR (KBr) $v_{\max } / \mathrm{cm}^{-1} 3058.9$ (aromatic $\mathrm{CH}$ ), 2962.5, 2873.7 (aliphatic $\mathrm{CH}), 1751.2(\mathrm{C}=\mathrm{O}), 1643.2(\mathrm{C}=\mathrm{N}), 1593.1(\mathrm{C}=\mathrm{C}) ;{ }^{1} \mathrm{H}$ NMR (DMSO-d $\left.\mathrm{d}_{6}\right) \delta 1.64(\mathrm{~s}, 3 \mathrm{H}$, $\mathrm{CH}_{3}$-imidazolinone), 5.42 (s, 1H, 4H-pyrazole), 7.02-7.71 (m, 17H, ArH's); MS m/z (\%): 491 (M $\left.\mathrm{M}^{+}, 30\right), 463$ (17), 371 (14), 370 (13), 118 (100), 77 (75); Anal. Calcd. for $\mathrm{C}_{28} \mathrm{H}_{21} \mathrm{~N}_{5} \mathrm{O}_{4}: \mathrm{C}, 68.42 ; \mathrm{H}, 4.31$; N, 14.25. Found: $\mathrm{C}$, $69.01 ; \mathrm{H}, 4.29 ; \mathrm{N}, 14.08 \%$.

8-methyl-7-(4-methylphenyl)-1,3,4-triphenyl-1,2,7,9-tetrazaspiro[4,4]nona-2,8-dien-6-one 4e. Off white crystals, yield 65\%; mp $214-216{ }^{\circ} \mathrm{C}$ (tetrahydrofuran); IR (KBr) $v_{\max } / \mathrm{cm}^{-1}$ 3058.9, 3035.7 (aromatic CH), 2970.2, 2923.9, 2862.2 (aliphatic $\mathrm{CH}), 1747.4(\mathrm{C}=\mathrm{O}), 1647.1(\mathrm{C}=\mathrm{N}), 1596.9(\mathrm{C}=\mathrm{C}) ;{ }^{1} \mathrm{H}$ NMR (DMSO-d $\left.\mathrm{d}_{6}\right) \delta 1.65(\mathrm{~s}, 3 \mathrm{H}$, $\mathrm{CH}_{3}$-imidazolinone), 2.37 (s, 3H, $\mathrm{CH}_{3}-\mathrm{Ph}$ ), 5.62 (s, 1H, 4H-pyrazole), 7.15-7.82 (m, 19H, ArH's); MS m/z (\%): $470\left(\mathrm{M}^{+}, 37\right), 336(56), 132(69), 91(100)$; Anal. Calcd. for $\mathrm{C}_{31} \mathrm{H}_{26} \mathrm{~N}_{4} \mathrm{O}: \mathrm{C}, 79.12 ; \mathrm{H}, 5.57 ; \mathrm{N}, 11.91$. Found: $\mathrm{C}$, $79.08 ; \mathrm{H}, 5.60 ; \mathrm{N}, 12.00 \%$.

3-(4-chlorophenyl)-8-methyl-7-(4-methylphenyl)-1, 4-diphenyl-1, 2, 7, 9-tetrazaspiro[4, 4]nona-2,8-dien-6-one 4f. Off white crystals, yield $85 \%$; mp $186-188{ }^{\circ} \mathrm{C}$ (benzene/pet.ether $40-60{ }^{\circ} \mathrm{C}$ ); IR (KBr) $v_{\max } / \mathrm{cm}^{-1} 3031.9$ (aromatic CH), 2923.9, 2862.2 (aliphatic $\mathrm{CH}), 1755.1(\mathrm{C}=\mathrm{O}), 1643.2(\mathrm{C}=\mathrm{N}), 1596.9(\mathrm{C}=\mathrm{C}) ;{ }^{1} \mathrm{H}$ NMR $\left(\mathrm{DMSO}-\mathrm{d}_{6}\right)$ $\delta 1.67\left(\mathrm{~s}, 3 \mathrm{H}, \mathrm{CH}_{3}\right.$-imidazolinone), $2.38\left(\mathrm{~s}, 3 \mathrm{H}, \mathrm{CH}_{3}-\mathrm{Ph}\right), 5.56(\mathrm{~s}, 1 \mathrm{H}, 4 \mathrm{H}$-pyrazole), 7.09-7.61 (m, $18 \mathrm{H}, \mathrm{ArH}$ 's); MS m/z (\%): $507\left(\mathrm{M}^{+}+2,4\right), 505\left(\mathrm{M}^{+}, 12\right), 504(31), 370$ (46), 132 (75), 91 (100), 77 (21); Anal. Calcd. for $\mathrm{C}_{31} \mathrm{H}_{25} \mathrm{ClN}_{4} \mathrm{O}: \mathrm{C}, 73.73 ; \mathrm{H}, 4.99 ; \mathrm{Cl}, 7.02 ; \mathrm{N}, 11.09$. Found: $\mathrm{C}, 37.71 ; \mathrm{H}, 4.95 ; \mathrm{N}, 11.10 \%$.

3-(4-Fluorophenyl)-8-methyl-7-(4-methylphenyl)-1-(4-nitrophenyl)-4-phenyl-1,2,7,9-tetrazaspiro[4,4]nona-2,8-d ien-6-one 4g. Canarian yellow crystals, yield 52\%; mp $260-262{ }^{\circ} \mathrm{C}$ (benzene/ethanol); IR (KBr) $v_{\max } / \mathrm{cm}^{-1}$ 3062.7 (aromatic CH), 2920.0, 2877.6 (aliphatic $\mathrm{CH}), 1751.2(\mathrm{C}=\mathrm{O}), 1643.2(\mathrm{C}=\mathrm{N}), 1596.9(\mathrm{C}=\mathrm{C}) ;{ }^{1} \mathrm{H}$ NMR (DMSO-d $\left.\mathrm{d}_{6}\right) \delta 1.72\left(\mathrm{~s}, 3 \mathrm{H}, \mathrm{CH}_{3}\right.$-imidazolinone), $2.38\left(\mathrm{~s}, 3 \mathrm{H}, \mathrm{CH}_{3}-\mathrm{Ph}\right), 5.63(\mathrm{~s}, 1 \mathrm{H}, 4 \mathrm{H}$-pyrazole), 7.13-8.27 (m, $17 \mathrm{H}, \mathrm{ArH}$ 's); ${ }^{13} \mathrm{C}$ NMR (DMSO-d 6$) \delta 15.440\left(\mathrm{CH}_{3}\right.$-imidazolinone), $20.659\left(\mathrm{CH}_{3}-\mathrm{Ph}\right), 63.266$ (CH-pyrazole), 89.550 (C-spiro), 113.258, 115.791-115.501 (d, J = 21.7 Hz, C-o-F), 125.889, 126.698, 127.228, 128.128-128.082 (d, J = 3.5 Hz, C-p-F), 128.212, 129.543-129.429 (d, J = 8.6 Hz, C-m-F), 129.74 (9C, ArCH's), 129.894, 130.154, 132.271, 139.126, 140.057, 148.018, 152.108, 162.781, 164.483-161.187 (d, J = 248.6 Hz, C-F) (9C, C=N, ArC's), 178.437 (CO); MS m/z (\%): 533 (M+, 47), 505 (38), 504 (37), 399 (40), 353 (21), 132 (100), 107 (65), 91 (82); Anal. Calcd. for $\mathrm{C}_{31} \mathrm{H}_{24} \mathrm{FN}_{5} \mathrm{O}_{3}$ : C, 69.78; H, 4.53; N, 13.13. Found: $\mathrm{C}, 69.83 ; \mathrm{H}, 4.55$; N, $13.05 \%$. 
8-Methyl-7-(4-methylphenyl)-1-(4-nitrophenyl)-3,4-diphenyl-1,2,7,9-tetrazaspiro[4,4]nona-2,8-dien-6-one 4h. Canarian yellow crystals, yield $87 \%$; mp $280-282{ }^{\circ} \mathrm{C}$ (tetrahydrofuran); IR (KBr) $v_{\max } / \mathrm{cm}^{-1} 3062.7,3035.7$ (aromatic CH), 2862.2 (aliphatic $\mathrm{CH}), 1751.2(\mathrm{C}=\mathrm{O}), 1643.2(\mathrm{C}=\mathrm{N}), 1589.2(\mathrm{C}=\mathrm{C}) ;{ }^{1} \mathrm{H}$ NMR $\left(\mathrm{DMSO}-\mathrm{d}_{6}\right) \delta 1.70$ (s, 3H, $\mathrm{CH}_{3}$-imidazolinone), 2.39 (s, 3H, $\left.\mathrm{CH}_{3}-\mathrm{Ph}\right), 5.54$ (s, 1H, 4H-pyrazole), 7.12-8.29 (m, 18H, ArH's); MS m/z (\%): $515\left(\mathrm{M}^{+}, 46\right), 514$ (39), 487 (46), 486 (39), 381 (40), 380 (36), 335 (22), 132 (99), 91 (100); Anal. Calcd. for $\mathrm{C}_{31} \mathrm{H}_{25} \mathrm{~N}_{5} \mathrm{O}_{3}$ : C, 72.22; H, 4.89; N, 13.58. Found: C, 71.94; H, 4.85; N, 13.59\%.

3-(2-Furyl)-8-Methyl-7-(4-methylphenyl)-1-(4-nitrophenyl)-4-phenyl-1,2,7,9-tetrazaspiro[4,4]nona-2,8-dien-6-one 4i. Orange crystals, yield 62\%; mp $270-272{ }^{\circ} \mathrm{C}$ (dioxane); IR (KBr) $v_{\max } / \mathrm{cm}^{-1} 3112.9,3062.7$ (aromatic $\mathrm{CH}$ ), 2966.3, 2869.9 (aliphatic $\mathrm{CH}), 1751.2(\mathrm{C}=\mathrm{O}), 1643.2(\mathrm{C}=\mathrm{N}), 1589.2(\mathrm{C}=\mathrm{C}) ;{ }^{1} \mathrm{H}$ NMR (DMSO-d $)_{6} \delta 1.69(\mathrm{~s}, 3 \mathrm{H}$, $\mathrm{CH}_{3}$-imidazolinone), 2.37 (s, 3H, $\left.\mathrm{CH}_{3}-\mathrm{Ph}\right), 5.62$ (s, 1H, 4H-pyrazole), 7.14-8.19 (m, 16H, ArH's); MS m/z (\%): $505\left(\mathrm{M}^{+}, 41\right), 504$ (41), 476 (29), 371 (20), 370 (15), 132 (100), 107 (43), 91 (82); Anal. Calcd. for $\mathrm{C}_{29} \mathrm{H}_{23} \mathrm{~N}_{5} \mathrm{O}_{4}$ : C, 68.90; H, 4.59; N, 13.85. Found: C, 69.00; H, 4.60; N, 13.78\%.

7-(2-methoxyphenyl)-8-methyl-1,3,4-triphenyl-1,2,7,9-tetrazaspiro[4,4]nona-2,8-dien-6-one 4j. White crystals, yield 80\%; mp $202-204{ }^{\circ} \mathrm{C}$ (tetrahydrofuran/pet.ether $\left.40-60{ }^{\circ} \mathrm{C}\right)$; IR (KBr) $v_{\max } / \mathrm{cm}^{-1} 3058.9$ (aromatic CH), 2943.2, 2893.0 (aliphatic $\mathrm{CH}), 1747.4(\mathrm{C}=\mathrm{O}), 1643.2(\mathrm{C}=\mathrm{N}), 1596.9(\mathrm{C}=\mathrm{C}) ;{ }^{1} \mathrm{H}$ NMR (DMSO-d $\mathrm{d}_{6} \delta 1.59(\mathrm{~s}, 3 \mathrm{H}$, $\mathrm{CH}_{3}$-imidazolinone), 3.89 (s, 3H, $\mathrm{CH}_{3} \mathrm{O}-\mathrm{Ph}$ ), 5.40 (s, 1H, 4H-pyrazole), 6.94-7.60 (m, 19H, ArH's); ${ }^{13} \mathrm{C} \mathrm{NMR}$ $\left(\mathrm{DMSO}_{6}\right) \delta 14.555\left(\mathrm{CH}_{3}\right.$-imidazolinone), $56.174\left(\mathrm{OCH}_{3}\right), 63.750(\mathrm{CH}$-pyrazole $), 90.526(\mathrm{C}$-spiro $), 112.701$, $114.723,120.823,121.239,126.701,127.518,27.720,127.834,128.334,128.784,128.914,129.036,129.185$ (13C, CHArC's), 130.249, 131.096, 132.565, 143.296, 149.07, 154.629, 161.873, (7C, C=N, ArC's), 179.574 (CO); MS m/z (\%): $486\left(\mathrm{M}^{+}, 54\right), 427$ (27), 336 (77), 148 (71), 91 (76), 77 (100); Anal. Calcd. for $\mathrm{C}_{31} \mathrm{H}_{26} \mathrm{~N}_{4} \mathrm{O}_{2}$ : C, 76.52; H, 5.39; N, 11.51. Found: C, 76.61; H, 5.41; N, 11.50\%.

3-(4-Cholrophenyl)-7-(2-methoxyphenyl)-8-methyl-1, 4-diphenyl-1,2,7,9-tetrazaspiro[4,4]nona-2,8-dien-6-one 4k. White crystals, yield 97\%; mp $234-236{ }^{\circ} \mathrm{C}$ (benzene/ethanol); IR (KBr) $v_{\max } / \mathrm{cm}^{-1} 3066.6,3031.9$ (aromatic CH), 2954.7, 2846.7 (aliphatic CH), $1751.2(\mathrm{C}=\mathrm{O}), 1647.1(\mathrm{C}=\mathrm{N}), 1600.8(\mathrm{C}=\mathrm{C}) ;{ }^{1} \mathrm{H}$ NMR (DMSO-d $\mathrm{d}_{6} \delta 1.64(\mathrm{~s}, 3 \mathrm{H}$, $\mathrm{CH}_{3}$-imidazolinone), 3.88 (s, 3H, $\mathrm{CH}_{3} \mathrm{O}-\mathrm{Ph}$ ), 5.43 (s, 1H, 4H-pyrazole), 7.14-7.65 (m, 18H, ArH's); MS m/z (\%): $523\left(\mathrm{M}^{+}+2,6\right), 521\left(\mathrm{M}^{+}, 20\right), 461(28), 370$ (65), 148 (84), 91 (84), 77 (100); Anal. Calcd. for $\mathrm{C}_{31} \mathrm{H}_{25} \mathrm{ClN}_{4} \mathrm{O}_{2}: \mathrm{C}_{\text {, }}$ 71.46; H, 4.48; Cl, 6.80; N, 10.75. Found: C, 71.52; H, 4.51; Cl, 6.80; N, 10.83\%.

3-(2,4-Dicholrophenyl)-7-(2-methoxyphenyl)-8-methyl-1-(4-nitrophenyl)-4-phenyl-1,2,7,9-tetrazaspiro[4,4]nona -2,8-dien-6-one 4l. Yellow crystals, yield 58\%; mp $268-270{ }^{\circ} \mathrm{C}$ (dioxane/ethanol); IR (KBr) $v_{\max } / \mathrm{cm}^{-1} 3058.9$ (aromatic CH), 2927.7, 2835.2 (aliphatic $\mathrm{CH}), 1755.1(\mathrm{C}=\mathrm{O}), 1643.2(\mathrm{C}=\mathrm{N}), 1593.1(\mathrm{C}=\mathrm{C}) ;{ }^{1} \mathrm{H}$ NMR $\left(\mathrm{DMSO}-\mathrm{d}_{6}\right)$ $\delta 1.62$ (s, 3H, $\mathrm{CH}_{3}$-imidazolinone), 3.87 (s, 3H, $\mathrm{CH}_{3} \mathrm{O}-\mathrm{Ph}$ ), 5.41 (s, 1H, 4H-pyrazole), 7.02-7.91 (m, 16H, ArH's); MS m/z (\%): $602\left(\mathrm{M}^{+}+2,7\right), 600\left(\mathrm{M}^{+}, 15\right), 570$ (20), 540 (24), 449 (17), 403 (13), 123 (100), 77 (56); Anal. Calcd. for $\mathrm{C}_{31} \mathrm{H}_{23} \mathrm{Cl}_{2} \mathrm{~N}_{5} \mathrm{O}_{4}$ : C, 62.01; H, 3.86; Cl, N, 11.81. Found: C, 62.00; H, 3.91; Cl; N, 11.76\%.

3-(2-Furyl)-7-(2-methoxyphenyl)-8-methyl-1-(4-nitrophenyl)-4-phenyl-1,2,7,9-tetrazaspiro[4,4]nona-2,8-dien-6one 4m. Yellow crystals, yield 57\%; mp $214-216{ }^{\circ} \mathrm{C}$ (tetrahydrofuran/pet.ether $\left.40-60{ }^{\circ} \mathrm{C}\right) ; \mathrm{IR}(\mathrm{KBr}) v_{\max } / \mathrm{cm}^{-1}$ 3082.0, 3043.5 (aromatic CH), 2939.3, 2842.9 (aliphatic $\mathrm{CH}), 1751.2(\mathrm{C}=\mathrm{O}), 1627.8(\mathrm{C}=\mathrm{N}), 1596.9(\mathrm{C}=\mathrm{C}) ;{ }^{1} \mathrm{H}$ NMR (DMSO-d 6$) \delta 1.64\left(\mathrm{~s}, 3 \mathrm{H}, \mathrm{CH}_{3}\right.$-imidazolinone), 3.73 (s, 3H, $\left.\mathrm{CH}_{3} \mathrm{O}-\mathrm{Ph}\right), 5.40$ (s, 1H, 4H-pyrazole), 6.88-7.74 (m, 16H, ArH's); MS m/z (\%): 522 (M+1, 40), 461 (33), 370 (58), 148 (92), 91 (78), 77 (100); Anal. Calcd. for $\mathrm{C}_{29} \mathrm{H}_{23} \mathrm{~N}_{5} \mathrm{O}_{5}$ : C, 66.79; H, 4.45; N, 13.43. Found: C, 66.81; H, 4.48; N, 13.44\%.

7-(3-Methoxyphenyl)-8-methyl-1,3,4-triphenyl-1,2,7,9-tetrazaspiro[4,4]nona-2,8-dien-6-one 4n. Off white crystals, yield 94\%; mp $232-234{ }^{\circ} \mathrm{C}$ (tetrahydrofuran/methanol); IR (KBr) $v_{\max } / \mathrm{cm}^{-1} 3058.9,3028.0$ (aromatic $\mathrm{CH}), 2962.5,2835.2$ (aliphatic $\mathrm{CH}), 1759.0(\mathrm{C}=\mathrm{O}), 1647.1(\mathrm{C}=\mathrm{N}), 1596.9(\mathrm{C}=\mathrm{C}) ;{ }^{1} \mathrm{H}$ NMR $\left(\mathrm{DMSO}-\mathrm{d}_{6}\right) \delta 1.68(\mathrm{~s}$, $3 \mathrm{H}, \mathrm{CH}_{3}$-imidazolinone), 3.81 (s, 3H, $\left.\mathrm{CH}_{3} \mathrm{O}-\mathrm{Ph}\right), 5.49$ (s, 1H, 4H-pyrazole), 7.14-7.63 (m, 19H, ArH's); MS m/z (\%): $487\left(\mathrm{M}^{+}+1,34\right), 381$ (40), 193 (14), 132 (83), 91 (100), 77 (49); Anal. Calcd. for $\mathrm{C}_{31} \mathrm{H}_{26} \mathrm{~N}_{4} \mathrm{O}_{2}$ : C, 76.52; $\mathrm{H}$, $5.39 ; \mathrm{N}, 11.51$. Found: C, 76.66; H, 5.40; N, 11.55\%.

3-(4-Chlorophenyl)-7-(3-methoxyphenyl)-8-methyl-1,4-diphenyl-1,2,7,9-tetrazaspiro[4,4]nona-2,8-dien-6-one 4o. Off white crystals, yield $86 \%$; mp $198-200{ }^{\circ} \mathrm{C}$ (tetrahydrofuran/pet.ether $40-60{ }^{\circ} \mathrm{C}$ ); IR $(\mathrm{KBr}) v_{\max } / \mathrm{cm}^{-1}$ 3043.5 (aromatic CH), 2935.5, 2835.5 (aliphatic $\mathrm{CH}), 1751.2(\mathrm{C}=\mathrm{O}), 1639.4(\mathrm{C}=\mathrm{N}), 1596.9(\mathrm{C}=\mathrm{C}) ;{ }^{1} \mathrm{H} \mathrm{NMR}$ (DMSO-d $\left.{ }_{6}\right) \delta 1.69$ (s, 3H, $\mathrm{CH}_{3}$-imidazolinone), 3.82 (s, 3H, $\left.\mathrm{CH}_{3} \mathrm{O}-\mathrm{Ph}\right), 5.39$ (s, 1H, 4H-pyrazole), 7.08-7.60 (m, $18 \mathrm{H}, \mathrm{ArH}$ 's); ${ }^{13} \mathrm{C}$ NMR (DMSO-d 6$) \delta 15.242\left(\mathrm{CH}_{3}\right.$-imidazolinone), $55.346\left(\mathrm{OCH}_{3}\right), 62.423(\mathrm{CH}-\mathrm{pyrazole})$, 91.049 (C-spiro), 112.869, 114.571, 115.169, 119.099, 121.418, 127.731, 127.876, 127.921, 128.124, 128.357, 129.044, 129.780 (12C, CHArC's), 130.398, 132.519, 133.377, 133.457, 143.261, 148.682, 159.863, 161.183 (8C, C=N, ArC's), 178.708 (CO); MS m/z (\%): $522\left(\mathrm{M}^{+}+2,25\right), 521,(41), 520\left(\mathrm{M}^{+}, 73\right), 492$ (42), 491 (41), 370 
(79), 148 (97), 107 (51), 91 (95), 77 (100); Anal. Calcd. for $\mathrm{C}_{31} \mathrm{H}_{25} \mathrm{ClN}_{4} \mathrm{O}_{2}: \mathrm{C}, 71.46 ; \mathrm{H}, 4.84 ; \mathrm{Cl}, 6.80 ; \mathrm{N}, 10.75$. Found: C, 71.52; H, 4.86; Cl, 6.80; N, 10.75\%.

3-(4-Fluorophenyl)-7-(3-methoxyphenyl)-8-methyl-1-(4-nitrophenyl)-4-phenyl-1,2,7,9-tetrazaspiro[4,4]nona-2,8 -dien-6-one 4p. Yellow crystals, yield 59\%; mp $239-241{ }^{\circ} \mathrm{C}$ (tetrahydrofuran/ethanol); IR $(\mathrm{KBr}) v_{\max } / \mathrm{cm}^{-1}$ 3078.2 (aromatic $\mathrm{CH}$ ), 2842.9 (aliphatic $\mathrm{CH}), 1751.2(\mathrm{C}=\mathrm{O}), 1651.0(\mathrm{C}=\mathrm{N}), 1596.9(\mathrm{C}=\mathrm{C}) ;{ }^{1} \mathrm{H}$ NMR $\left(\mathrm{DMSO}-\mathrm{d}_{6}\right)$ $\delta 1.69$ (s, 3H, $\mathrm{CH}_{3}$-imidazolinone), 3.82 (s, 3H, $\left.\mathrm{CH}_{3} \mathrm{O}-\mathrm{Ph}\right), 5.39$ (s, 1H, 4H-pyrazole), 7.08-7.60 (m, 17H, ArH's); MS m/z (\%): $549\left(\mathrm{M}^{+}, 50\right), 521$ (48), 399 (45), 353 (22), 148 (100), 90 (38), 77 (100); Anal. Calcd. for $\mathrm{C}_{31} \mathrm{H}_{24} \mathrm{FN}_{5} \mathrm{O}_{4}: \mathrm{C}, 67.75 ; \mathrm{H}, 4.40 ; \mathrm{N}, 12.74$. Found: $\mathrm{C}, 67.80 ; \mathrm{H}, 4.41 ; \mathrm{N}, 12.75 \%$.

7-(4-Ethoxyphenyl)-8-methyl-1,3,4-triphenyl-1,2,7,9-tetrazaspiro[4,4]nona-2,8-dien-6-one 4q. Off white crystals, yield 74\%; mp $220-222{ }^{\circ} \mathrm{C}$ (benzene/ethanol); IR (KBr) $v_{\max } / \mathrm{cm}^{-1} 3077.5$ (aromatic $\mathrm{CH}$ ), 2977.9, 2873.7 (aliphatic $\mathrm{CH}), 1747.4(\mathrm{C}=\mathrm{O}), 1647.1(\mathrm{C}=\mathrm{N}), 1596.9(\mathrm{C}=\mathrm{C}) ;{ }^{1} \mathrm{H}$ NMR $\left(\right.$ DMSO-d $\left._{6}\right) \delta 1.33(\mathrm{t}, 3 \mathrm{H}, \mathrm{J}=7.2 \mathrm{~Hz}$, $\mathrm{OCH}_{2} \mathbf{C H}_{3}$ ), 1.64 (s, 3H, $\mathrm{CH}_{3}$-imidazolinone), 4.05 (q, 2H, J = 7.2 Hz, $\mathrm{OCH}_{2} \mathrm{CH}_{3}$ ), 5.39 (s, 1H, 4H-pyrazole), 6.95-7.66 (m, 19H, ArH's); MS m/z (\%): 500 (M+ 44), 399 (46), 336 (44), 323 (44), 192 (24), 148 (68), 91 (68), 77 (100); Anal. Calcd. for $\mathrm{C}_{32} \mathrm{H}_{28} \mathrm{~N}_{4} \mathrm{O}_{2}: \mathrm{C}, 76.78 ; \mathrm{H}, 5.64 ; \mathrm{N}, 11.19$. Found: $\mathrm{C}, 76.82 ; \mathrm{H}, 5.66 ; \mathrm{N}, 11.22 \%$.

3-(4-Chlorophenyl)-7-(4-ethoxyphenyl)-8-methyl-1,4-diphenyl-1,2,7,9-tetrazaspiro[4,4]nona-2,8-dien-6-one 4r. Off white crystals, yield 94\%; mp $191-193{ }^{\circ} \mathrm{C}$ (tetrahydrofuran/ethanol); IR (KBr) $v_{\max } / \mathrm{cm}^{-1} 3039.6$ (aromatic $\mathrm{CH}), 2985.6,2923.9,2869.9$ (aliphatic $\mathrm{CH}), 1751.2(\mathrm{C}=\mathrm{O}), 1647.1(\mathrm{C}=\mathrm{N}), 1596.9(\mathrm{C}=\mathrm{C}) ;{ }^{1} \mathrm{H}$ NMR $\left(\mathrm{DMSO}-\mathrm{d}_{6}\right)$ $\delta 1.33\left(\mathrm{t}, 3 \mathrm{H}, \mathrm{J}=7.2 \mathrm{~Hz}, \mathrm{OCH}_{2} \mathbf{C H}_{3}\right), 1.63$ (s, 3H, $\mathrm{CH}_{3}$-imidazolinone), 4.06 (q, $\left.2 \mathrm{H}, \mathrm{J}=7.2 \mathrm{~Hz}, \mathrm{OCH}_{2} \mathrm{CH}_{3}\right), 5.41$ (s, 1H, 4H-pyrazole), 6.96-7.61 (m, 18H, ArH's); ${ }^{13} \mathrm{C}$ NMR (DMSO-d $\left.{ }_{6}\right) \delta 14.475\left(\mathrm{CH}_{3}\right.$-ethyl), 15.257 ( $\mathrm{CH}_{3}$-imidazolinone), 62.789 (CH-pyrazole), 63.499 ( $\mathrm{CH}_{2}$-ethyl), 91.030 (C-spiro), 115.166, 115.372, 121.452, 124.886, 127.850, 127.983, 128.269, 128.399, 128.456, 128.492 (10C, CHArC's), 129.177, 129.925, 132.591, 133.526, 143.425, 148.632, 158.886, 161.744 (8C, C=N, ArC's), 179.310 (CO); MS m/z (\%): $535\left(\mathrm{M}^{+}, 24\right), 534$ $\left(\mathrm{M}^{+}-1,55\right), 506$ (39), 370 (75), 162 (83), 137 (85), 91 (100), 77 (48); Anal. Calcd. for $\mathrm{C}_{32} \mathrm{H}_{27} \mathrm{ClN}_{4} \mathrm{O}_{2}$ : C, 71.83 ; $\mathrm{H}, 5.09 ; \mathrm{Cl}, 6.63 ; \mathrm{N}, 10.47$. Found: $\mathrm{C}, 71.90 ; \mathrm{H}, 5.12 ; \mathrm{Cl}, 6.61 ; \mathrm{N}, 10.51 \%$.

3-(2,4-Dichlorophenyl)-7-(4-ethoxyphenyl)-8-methyl-1-(4-nitrophenyl)-4-phenyl-1,2,7,9-tetrazaspiro[4,4]nona-2 ,8-dien-6-one 4s. Yellow crystals, yield 56\%; mp 298 - $300{ }^{\circ} \mathrm{C}$ (dimethylsulfoxide/ethanol); IR (KBr) $v_{\max } / \mathrm{cm}^{-1}$ 3074.3 (aromatic CH), 2981.7, 2935.5, 2885.3 (aliphatic $\mathrm{CH}), 1751.2(\mathrm{C}=\mathrm{O}), 1647.1(\mathrm{C}=\mathrm{N}), 1589.2(\mathrm{C}=\mathrm{C}) ;{ }^{1} \mathrm{H}$ NMR (DMSO-d $\left.)_{6}\right) \delta 1.34\left(\mathrm{t}, 3 \mathrm{H}, \mathrm{J}=7.1 \mathrm{~Hz}, \mathrm{OCH}_{2} \mathbf{C H}_{3}\right), 1.65$ (s, 3H, $\mathrm{CH}_{3}$-imidazolinone), 4.03 (q, 2H, J = 7.1 $\left.\mathrm{Hz}, \mathrm{OCH}_{2} \mathrm{CH}_{3}\right), 5.48$ (s, 1H, 4H-pyrazole), 7.01-7.93 (m, 16H, ArH's); MS m/z (\%): $616\left(\mathrm{M}^{+}+2,8\right), 614(12)$, 584 (24), 448 (14), 403 (10), 162 (50), 137 (100), 90 (18); Anal. Calcd. for $\mathrm{C}_{32} \mathrm{H}_{25} \mathrm{Cl}_{2} \mathrm{~N}_{5} \mathrm{O}_{4}$ : C, 62.55; H, 4.10; Cl, 11.54; N, 11.40. Found: C, 62.58; H, 4.08; Cl, 11.57; N, $11.43 \%$.

7-(4-ethoxyphenyl)-3-(2-furyl)-8-methyl-1-(4-nitrophenyl)-4-phenyl-1,2,7,9-tetrazaspiro[4,4]nona-2,8-dien-6-on e 4t. Orange crystals, yield 53\%; mp $227-229{ }^{\circ} \mathrm{C}$ (benzene); IR (KBr) $v_{\max } / \mathrm{cm}^{-1} 3066.6$ (aromatic $\mathrm{CH}$ ), 2989.5, 2908.5, 2869.9 (aliphatic CH), $1751.2(\mathrm{C}=\mathrm{O}), 1639.4(\mathrm{C}=\mathrm{N}), 1593.1(\mathrm{C}=\mathrm{C}) ;{ }^{1} \mathrm{H}$ NMR $\left(\mathrm{DMSO}-\mathrm{d}_{6}\right) \delta 1.32(\mathrm{t}, 3 \mathrm{H}$,

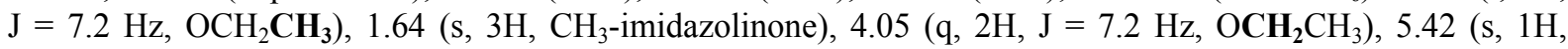
4H-pyrazole), 7.03-7.71 (m, 16H, ArH's); MS m/z (\%): 535 (M+, 46), 534 (40), 507 (33), 162 (70), 137 (100), 134 (27), 65 (39); Anal. Calcd. for $\mathrm{C}_{30} \mathrm{H}_{25} \mathrm{~N}_{5} \mathrm{O}_{5}$ : C, 67.28; H, 4.71; N, 13.08. Found: C, 67.30; H, 4.73; N, $13.10 \%$.

7-(2-Methoxyphenyl)-4-(4-methoxyphenyl)-8-methyl-1,3-diphenyl-1,2,7,9-tetrazaspiro[4,4]nona-2,8-dien-6-one 4u. Off white crystals, yield 95\%; mp $192-194{ }^{\circ} \mathrm{C}$ (tetrahydrofuran/ethanol); IR (KBr) $v_{\max } / \mathrm{cm}^{-1} 3062.0,3035.7$ (aromatic CH), 2974.0, 2939.3, 2839.0 (aliphatic $\mathrm{CH}), 1751.2(\mathrm{C}=\mathrm{O}), 1643.2(\mathrm{C}=\mathrm{N}), 1596.9(\mathrm{C}=\mathrm{C}) ;{ }^{1} \mathrm{H}$ NMR (DMSO-d $\mathrm{d}_{6}$ ) $\delta 1.64$ (s, 3H, $\mathrm{CH}_{3}$-imidazolinone), 3.73, 3.80 (two s, 6H, two $\mathrm{CH}_{3} \mathrm{O}-\mathrm{Ph}$ ), 5.30 (s, $1 \mathrm{H}, 4 \mathrm{H}$-pyrazole), 6.88-7.60 (m, 18H, ArH's); ${ }^{13} \mathrm{C}$ NMR (DMSO-d $\left.\mathrm{d}_{6}\right) \delta 14.635$ ( $\mathrm{CH}_{3}$-imidazolinone), 54.828, $55.606\left(2 \mathrm{C}, \mathrm{OCH}_{3}\right)$, 62.427 (CH-pyrazole), 90.366 (C-spiro), 113.033, 114.525, 114.849, 120.735, 120.922, 124.161, 124.579, 126.499, 128.117, 128.670, 128.822, 128.933 (12C, CHArC's), 129.063, 131.054, 131.218, 143.185, 149.105, 154.965, 158.661, 160.897 (8C, C=N, ArC's), $179.459(\mathrm{CO}) ; \mathrm{MS} \mathrm{m} / \mathrm{z}(\%): 516\left(\mathrm{M}^{+}, 79\right), 488(38), 456(28), 365$ (39), 366 (52), 148 (91), 91 (87), 77 (100); Anal. Calcd. for $\mathrm{C}_{32} \mathrm{H}_{28} \mathrm{~N}_{4} \mathrm{O}_{3}$ : C, 74.40; H, 5.46; N, 10.85. Found: C, $74.42 ; \mathrm{H}, 5.49 ; \mathrm{N}, 11.00 \%$.

3-(4-Chlorophenyl)-7-(2-methoxyphenyl)-4-(4-methoxyphenyl)-8-methyl-1-phenyl-1,2,7,9-tetrazaspiro[4,4]non a-2,8-dien-6-one 4v. Off white crystals, yield 82\%; mp 134 - $136{ }^{\circ} \mathrm{C}$ (benzene/ethanol); IR $(\mathrm{KBr}) v_{\max } / \mathrm{cm}^{-1}$ 3066.6, 3039.6 (aromatic CH), 2966.3, 2953.5, 2839.0 (aliphatic $\mathrm{CH}), 1751.2(\mathrm{C}=\mathrm{O}), 1643.2(\mathrm{C}=\mathrm{N}), 1600.8$ $(\mathrm{C}=\mathrm{C}) ;{ }^{1} \mathrm{H}$ NMR (DMSO-d $\left.\mathrm{d}_{6}\right) \delta 1.64\left(\mathrm{~s}, 3 \mathrm{H}, \mathrm{CH}_{3}\right.$-imidazolinone), 3.74, 3.82 (two s, $6 \mathrm{H}$, two $\mathrm{CH}_{3} \mathrm{O}-\mathrm{Ph}$ ), $5.32(\mathrm{~s}$, 1H, 4H-pyrazole), 6.93-7.59 (m, 17H, ArH's); MS m/z (\%): 551 (M+1, 6), 322 (56), 148 (100), 91 (30), 77 (65); 
Anal. Calcd. for $\mathrm{C}_{32} \mathrm{H}_{27} \mathrm{ClN}_{4} \mathrm{O}_{3}: \mathrm{C}, 69.75 ; \mathrm{H}, 4.94 ; \mathrm{Cl}, 6.43 ; \mathrm{N}, 10.17$. Found: $\mathrm{C}, 70.00 ; \mathrm{H}, 5.00 ; \mathrm{Cl}, 6.44 ; \mathrm{N}$, $10.21 \%$.

3-(2-Furyl)-7-(2-methoxyphenyl)-4-(4-methoxyphenyl)-8-methyl-1-(4-nitrophenyl)-1,2,7,9-tetrazaspiro[4,4]non a-2,8-dien-6-one 4w. Canarian yellow crystals, yield 54\%; mp $160-162{ }^{\circ} \mathrm{C}$ (tetrahydrofuran); IR (KBr) $v_{\max } / \mathrm{cm}^{-1}$ 3070.5, 3004.9 (aromatic CH), 2974.0, 2912.3, 2839.0 (aliphatic $\left.\mathrm{CH}\right), 1705.0(\mathrm{C}=\mathrm{O}), 1643.2(\mathrm{C}=\mathrm{N})$, $1596.9(\mathrm{C}=\mathrm{C}) ;{ }^{1} \mathrm{H}$ NMR (DMSO-d $\left.\mathrm{d}_{6}\right) \delta 1.66$ (s, 3H, $\mathrm{CH}_{3}$-imidazolinone), 3.75, 3.83 (two s, 6H, two $\mathrm{CH}_{3} \mathrm{O}-\mathrm{Ph}$ ), 5.36 (s, 1H, 4H-pyrazole), 6.91-7.62 (m, 15H, ArH's); MS m/z (\%): 551 (M+, 24), 391 (53), 390 (41), 374 (27), 267 (28), 200 (20), 123 (100), 92 (29), 77 (17); Anal. Calcd. for $\mathrm{C}_{30} \mathrm{H}_{25} \mathrm{~N}_{5} \mathrm{O}_{6}$ : C, 65.33; H, 4.57; N, 12.70. Found: C, 65.36; H, 4.60; N, 12.73\%.

\subsection{In Vitro Antimicrobial Activity Screening}

For the in vitro antimicrobial activity, a stock solution of the synthesized compound $(1000 \mu \mathrm{g} / \mathrm{mL})$ in DMSO was prepared and $30 \mu \mathrm{l}$ of graded dilutions (Table 1) of the tested compounds were incorporated in a cavity (depth $3 \mathrm{~mm}$, diameter $4 \mathrm{~mm}$ ) made in the center of the petri plate which corresponding to $30 \mu \mathrm{g}, 15 \mu \mathrm{g}, 7.5 \mu \mathrm{g}$ and $3.75 \mu \mathrm{g}$. The plates were allowed to stand at room temperature for two hours for diffusion and then incubated. The organisms were grown on Muller Hinton's agar with sheep blood $(5 \%)$ at $37{ }^{\circ} \mathrm{C}$ for 24 hours. After incubation period, the clear zone around the wells was carefully measured in $\mathrm{mm}$ as inhibition zones. The absence of a clear zone around the well was taken as inactivity. The antibiotics Amoxycillin $(10 \mu \mathrm{g} / \mathrm{disc})$, Amikacin $(10 \mu \mathrm{g} / \mathrm{disc})$, Ceftriazone $(10 \mu \mathrm{g} / \mathrm{disc})$, and Ciprofloxacin $(30 \mu \mathrm{g} / \mathrm{disc})$ were used as references and dimethylsulphoxide (DMSO) as solvent and control. The investigation results are listed in Table 1.

\section{References}

Abunada, N. M., Hassaneen, H. M., M.Abusamaha, A. S., \& Miqdad, O. A. (2009). Synthesis and antimicrobial evaluation of some new pyrazole, pyrazoline and chromeno[3, 4-c]pyrazole derivatives. J. Braz. Chem. Soc., 20(5), 975-87. http://dx.doi.org/10.1590/S0103-50532009000500024

Atta-ur-Rahman, Choudhary, M. I., \& Thomsen, W. J. (2001). Bioassy techniques for drug development, harwood academic publishers. The Netherlands, 16, 22.

Aylward, J. B., \& Scott, F. L. (1969). Preparation and solvolysis of N-arylbenzohydrazonyl bromides. J. Chem. Soc. (B), 1080-84. http://dx.doi.org/10.1039/j29690001080

Bhattacharjya, G., Savitha, G., \& Tamanathan, G. (2005). C-H...O interactions are favoured in the crystal $\begin{array}{llllll}\text { structures of imidazolin-5-ones. } \quad J . & \text { Mol. } & \text { Stru., } & 752(1-3), & 98-103 .\end{array}$ http://dx.doi.org/10.1016/j.molstruc.2005.05.044

Chande, M. S., Verma, R. S., Barve, P. A., Khanwelkar, R. R., Vaidya, R. B., \& Ajaikumar, K. B. (2005). Facile synthesis of active antitubercular, cytotoxic and antibacterial agents: a Michael addition approach. Eur. J. Med. Chem., 40(11), 1143-48. http://dx.doi.org/10.1016/j.ejmech.2005.06.004

Desai, N. C., Bhavsar, A. M., \& Baldaniya, B. B. (2009). Synthesis and antimicrobial activity of 5-imidazolinone derivatives. Indian J. Pharm. Sci., 71(1), 90-94. http://dx.doi.org/10.4103/0250-474X.51953

Frank, R., Reich, M., Jostock, R., Bahrenberg, G., Schick, H., Henkel, B., \& Sonnenschein, H. (2008). Substituted spiro compounds and their use for producing pain-relief medicaments. US Pat. 20080269271 (App USPTO: 514278).

Giorgioni, G., Claudi, F., Ruggieri S., Ricciutelli, M., F.Palmieri, G., Di-Stefano, A., Sozio, P., S.Cerasa, L., Chiavoroli, A., Ferrante, C., Orlando, G., \& R. A. Glennon. (2010). Design, synthesis, and preliminary pharmacological evaluation of new imidazolinones as L-DOPA prodrugs. Bioorg. Med. Chem., 18(5), 1834-43. http://dx.doi.org/10.1016/j.bmc.2010.01.041

Godefroi, E. F., \& Platje, J. T. J. (1972). DL-1-(.alpha.-Methylbenzyl)-2-methylimidazole-5-carboxylate esters. Synthesis and pharmacological properties. J. Med. Chem., 15(3), 336-37. http://dx.doi.org/10.1021/jm00273a035

Hans, S., Robert, F., Reich, M., Ruth, O., Gregor, B., Fritz, T., \& Henkel, B. (2006). Substituted spiro compounds and their use for producing pain-relief drugs. Int. Pat. WO/2006/122769 (App No: PCT/EP2006/004651).

Harfenist, M., Soroko, E. F., \& Mckenzie, G. M. (1978). 2-(Alkoxyaryl)-2-imidazoline monoamine oxidase inhibitors with antidepressant activity. J. Med. Chem., 21(4), 405-9. http://dx.doi.org/10.1021/jm00202a021

Hassaneen, H. M., Daboun, H. A., Abdelhadi, H. A., \& Abdel-Reheim, N. A. (1995). Site selectivity and regiochemistry of nitrilimines. Cycloadditions to 1,3-diphenyl-2-thiono-4-imidazolidinone and its 
5-phenylmethylene derivatives. Phosphorus Sulfur and Silicon, 107(1-4), 269-73. http://dx.doi.org/10.1080/10426509508027942

Hegarty, A. F., \& Scott, F. L. (1966). The kinetics of bromination of hydrazones. J. Chem. Soc., (B), 672-75. http://dx.doi.org/10.1039/j29660000672

Hejiao, H., Huijuan, G., Erwei, L., Xingzhong, L., Yuguang, Z., Yongsheng, C., \& Decaspirones, F.-I. (2006). Bioactive secondary metabolites from the saprophytic fungus Helicoma viridis. J. Nat. Prod., 69(12), 1672-75. http://dx.doi.org/10.1021/np0603830

Hyeong-Beom, P., Hyun, J. N., Hee, H. J., Hoon, C. J., Hoon, C. J., Jung-Hyuck, C., Ho, Y. K., \& Chang-Hyun, O. (2007). Synthesis and In-Vitro activity of novel $1 \beta$-methylcarbapenems having spiro[2, 4]heptane moieties. Arch Pharm, 340(10), 530-37. http://dx.doi.org/10.1002/ardp.200700060

Islam, A. M., Khalil, A. M., \& AbdEl-Gawad, I. I. (1973). Reaction of 2-Aryl-4-arylmethylene-2-oxazolin-5-ones with amines. Aust. J. Chem., 26(4), 827-30. http://dx.doi.org/10.1071/CH9730827

Jolanta, O., \& Krzysztof, K. (2006). Synthesis and anticonvulsant properties of new N-phenylamino derivatives of 2-azaspiro[4, 4]nonane, 2-azaspiro[4,5]decane-1,3-dione and 3-cyclohexylpyrrolidine-2,5-dione. Part IV. Acta. Pol. Pharm., 6(2) 3, 101-8.

Jolanta, O., Krzysztof, K., \& Ewa, T. (2006). Impact of aromatic substitution on the anticonvulsant activity of new N-(4-arylpiperazin-1-yl)-alkyl-2-azaspiro[4,5]decane-1,3-dione derivatives. Pharmacol Rep., 58(2), 207-14.

Karabasanagouda T., Airody, V. A., \& Girisha, M. (2009). Synthesis of some new pyrazolines and isoxazoles carrying 4-methylthiopphenyl moiety as potentioal analgesic and anti-inflammatory agents. Indian J. Chem., 48B, 430-437.

Korgaokar, S. S., Patil, P. H., Shah, M. J., \& Parekh, H. H. (1996). Studies on pyrazolines: preparation and antimicrobial activity of 3-(p-chlorophenylsulphonamidophenyl)-5 aryl- 1H/acetyl pyrazolines. Indian J. Pharm. Sci., 58(6), 222-25.

Kreuder, W., Yu, N., \& Salbeck, J. (1999). Use of spiro compounds as LASER dyes. Int. Pat. WO/1999/040655 (App PCT/EP1999/000441).

Krzysztof, K., Jolanta, O., \& Malgorzata, D. (2008). Synthesis, physicochemical and anticonvulsant properties of new N-phenylamino derivatives of 2-azaspiro[4,4]nonane- and [4,5]decane-1,3-diones: Part V. Eur. J. Med. Chem., 43(1), 53-61.

Lindell, S., Sanft, U., \& Thönessen, M.-T. (2001). Heterocyclic spiro compounds as pesticides. Int. Pat. WO/2001/011968 (App PCT/EP2000/ 007851).

Lupo, D., J. Salbeck, Schenk, H., Stehlin, T., Stern, R., \& Wolf, A. (2008). Spiro compounds and their use as electroluminescence materials. US Pat. 5840217 (App USPTO: 08/417390).

Masakazu, F., Kenji, H., \& Jiro, K. (2001). Spiro compound, process for preparing the same and use thereof as drugs. Int. Pat. WO/2001/066546 (App PCT/JP2001/001793).

Miqdad, O. A., Abunada, N. M., \& Hassaneen, H. M. (2011). Regioselectivity of nitrilimines 1,3-dipolar cycloaddition: Novel synthesis of spiro[4,4]nona-2,8-dien-6-one derivatives. Heteroatom chemistry, 22(2), 131-36. http://dx.doi.org/10.1002/hc.20666

Nakao, K., Ikeda, K., Kurokawa, T., Togashi, Y., Umeuchi, H., Honda, T., Okano, K., \& Mochizuki, H. (2008). A selective kappa opioid receptor agonist, on scratching behavior in an animal model of atopic dermatitis. Nihon Shinkei Seishin Yakurigaku Zasshi Japanese Journal of Psychopharmacology, 28(2), 75-83.

Nauduri, D., \& B. Reddy, G. (1998). Antibacetrials and antimycotics: part 1: Synthesis and activity of 2-pyrazoline derivatives. Chem. Pharm. Bull. (Tokyo), 46(8), 1254-60.

Ozdemir, Z., Kandilici, H. B., Gumusel, B., Calis, U., \& ABilgin, A. (2007). Synthesis and studies on antidepressant and anticonvulsant activities of some 3-(2-furyl)-pyrazoline derivatives. Eur. J. Med. Chem., 42(3), 373-79. http://dx.doi.org/10.1016/j.ejmech.2006.09.006

Palaska, E., Aytemir, M., Uzbay, I., \& Erol, D. (2001). Synthesis and antidepressant activities of some 3,5-diphenyl-2-pyrazolines. Eur. J. Med. Chem., 36(6), 539-43. http://dx.doi.org/10.1016/S0223-5234(01)01243-0 
Pawar, M. J., Burungale, A. B., \& Karale, B. K. (2009). Synthesis and antimicrobial activity of spiro(chromeno[4,3-d][1,2,3]thiadiazole-4,1'-cyclohexanes), spiro(chromeno-[4,3-d][1,2,3]-selenadiazole-4,1'cyclohexanes) and (spiro-chroman-2,1'-cyclohexan-4-one)-5-spiro-4-acetyl-2-(acetylamino)- $\Delta 2-1,3,4-$ thiadiazoline compounds. ARKIVOC, (XIII), 97-107.

Rajendra P. Y., Lakshmana, R. A., Prasoona, L., Murali, K., \& Ravi, K. P. (2005). Synthesis and antidepressant activity of some 1,3,5-triphenyl-2-pyrazolines and 3-(2"-hydroxy naphthalen-1"-yl)-1,5-diphenyl-2-pyrazolines. Bioorg. Med. Chem. Lett., 15(22), 5030-34. http://dx.doi.org/10.1016/j.bmcl.2005.08.040

Ruhoglu, O., Ozdemir, Z., Calis, U., Gumusel, B., \& Bilgin, A. A. (2005). Synthesis of and pharmacological studies on the antidepressant and anticonvulsant activities of some 1,3,5-trisubstituted pyrazolines. Arzneimittelforschung, 55(8), 431-36.

Sarma, B. K., Manna, D., Minoura, M., \& G. Mugesh. (2010). Synthesis, structure, spirocyclization mechanism and Glutathione Peroxidase-like antioxidant activity of stable spirodiazaselenurane and spirodiazatellurane. $J$. Am. Chem. Soc., 132(15), 5364-74. URL: http://pubs.acs.org/doi/abs/10.1021/ja908080u

Shawali, A. S., Ezmirly, S. T., \& Bukhari, A. M. (1992). Nuclear magnetic resonance spectroscopy and the structures of the regioisomeric products of the cycloaddition of C-ethoxycarbonyl-N-arylnitrilimines to $\alpha$,

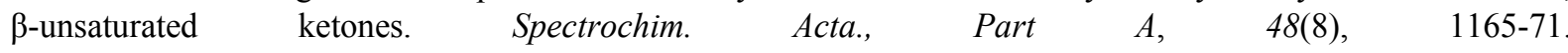
http://dx.doi.org/10.1016/0584-8539(92)80127-I

Shawali, A. S., \& Hassaneen, H. M. (1973). Reaction of carbanions of $\beta$-diketones and $\beta$-keto esters with hydrazidic bromides. Tetrahedron, 29(1), 121-24. http://dx.doi.org/10.1016/S0040-4020(01)99385-8

Shawali, A. S., Hassaneen, H. M., \& Ibrahim, H. A. (1990). Synthesis and cycloaddition reactions of N-aryl-2-furohydrazonyl chlorides. Arch Pharm Res, 13(2), 126-31. http://dx.doi.org/10.1007/BF02857788

Shimakawa, S., Yoshida, Y., \& Niki, E. (2003). Antioxidant action of lipophilic nitroxyl radical, cyclohexane-1-spiro-2'-(4'-oxyimidazolidine-1'-oxyl)-5'-spiro-1"-cyclohexane against peroxidation under hypoxic conditions. Lipids, 38(3), 225-31. http://dx.doi.org/10.1007/s11745-003-1055-3

Solankee, A., Kapadiya, K., Thakor, I., \& Patel, J. (2004). Synthesis and Antimicrobial Activity of 1-(4'-Trifluoro methylphenyl)-2-phenyl-4-(benzylidene/substituted Benzylidene/2' furylidene/2'-thienylidene) -imidazolin-5-ones. Asian J. Chem., 16(2), 917-20.

Taylor, E. C., \& Patel, H. H. (1992). Synthesis of pyrazolo[3,4-d]pyrimidine analogues of the potent agent N-4-2-2-amino-4(3H)-oxo-7H-pyrrolo [2,3-d]pyrimidin-5-yl)ethylbenzoyl-L-glutamic acid (LY231514). Tetrahedron, 48(37), 8089-100. http://dx.doi.org/10.1016/S0040-4020(01)80479-8

Thadhaney, B., Sain, D., Pernawat, G., \& Talesara, G. L. (2010). Synthesis and antimicrobial evaluation of ethoxyphthalimide derived from spiro[indole-3,5'-(1,3)thiazole(4,5-c)isoxazol]-2(1H)-ones via ring closure metathesis. Indian J. Chem., 49B, 368.

Van-Der-Sar, S., Blunt, J., \& Munro, M. (2006). Spiro-mamakone A: A unique relative of the spirobisnaphthalene class of compounds. Org. Lett., 8(10), 2059-61. http://dx.doi.org/10.1021/o1060434k

Wen-Liang, W., Tian-Jiao, Z., Hong-Wen, T., Zhen-Yu, L., Yu-Chun, F., Qian-Qun, G., \& Wei Ming, Z. (2007). Three novel, structurally unique spirocyclic alkaloids from the halotolerant $b-17$ fungal strain of aspergillus variecolor. Chem. Biodivers, 4(12), 2913-19. http://dx.doi.org/10.1002/cbdv.200790240

Wolkoff, P. (1975). A new method of preparing hydrazonyl halides. Can. J. Chem., 53(9), 1333-35. http://dx.doi.org/10.1139/v75-183

Young-Won, C., Angela, S., Bao-Ning, S., Quiwen, M., Hee-Byung, C., Soedarsono, R., Leonardus, K., Agus, R., Norman, F., Steven, S., \& Douglas, K. (2008). Potential anticancer activity of naturally occurring and semisynthetic derivatives of aculeatins a and b from amomum aculeatum. J. Nat. Prod., 71(3), 390-95. http://dx.doi.org/10.1021/np070584j 
Table 1. Antibacterial activity results of the synthesized compounds 4a-w

\begin{tabular}{|c|c|c|c|c|c|c|c|c|}
\hline \multicolumn{9}{|c|}{ Microorganisms $^{\mathrm{a}}$} \\
\hline \multicolumn{9}{|c|}{ Inhibition Zone diameter $(\mathrm{mm})^{\mathrm{d}}$} \\
\hline Comp. & $\begin{array}{l}\text { Conc. } \\
\mu \mathrm{g} / \mathrm{mL}\end{array}$ & E.c. & P.a. & V.s.. & C.s. & P.s. & S.s. & K.s. \\
\hline \multirow{4}{*}{$4 a$} & 1000 & 15 & 14 & - & 12 & 7 & 17 & 19 \\
\hline & 500 & 10 & 11 & - & 8 & - & 12 & 16 \\
\hline & 250 & 7 & 7 & - & - & - & 8 & 14 \\
\hline & 125 & - & - & - & - & - & - & 11 \\
\hline \multirow{4}{*}{$4 b$} & 1000 & 20 & 18 & 17 & 20 & 18 & 19 & 22 \\
\hline & 500 & 15 & 16 & 11 & 17 & 14 & 15 & 16 \\
\hline & 250 & 10 & 11 & - & 14 & 9 & 12 & 13 \\
\hline & 125 & 8 & - & - & 10 & - & 10 & 9 \\
\hline \multirow{4}{*}{$4 \mathrm{c}$} & 1000 & 12 & - & - & - & - & 17 & 23 \\
\hline & 500 & 10 & - & - & - & - & 13 & 19 \\
\hline & 250 & 8 & - & - & - & - & 10 & 17 \\
\hline & 125 & - & - & - & - & - & 8 & 14 \\
\hline \multirow{4}{*}{$4 d$} & 1000 & 15 & 20 & 16 & 20 & 17 & 14 & 19 \\
\hline & 500 & 14 & 18 & 10 & 17 & 12 & 11 & 16 \\
\hline & 250 & 10 & 12 & - & 13 & 9 & 8 & 11 \\
\hline & 125 & 8 & 8 & - & 9 & - & - & 8 \\
\hline \multirow{4}{*}{$4 \mathrm{e}$} & 1000 & 10 & - & - & 19 & 9 & 13 & 16 \\
\hline & 500 & 8 & - & - & 14 & - & 9 & 12 \\
\hline & 250 & - & - & - & 11 & - & 6 & 7 \\
\hline & 125 & - & - & - & 9 & - & - & - \\
\hline \multirow{4}{*}{$4 \mathrm{f}$} & 1000 & - & 13 & 20 & 20 & 14 & - & 12 \\
\hline & 500 & - & 9 & 14 & 14 & 10 & - & 9 \\
\hline & 250 & - & - & 9 & 10 & 7 & - & 6 \\
\hline & 125 & - & - & - & 8 & - & - & - \\
\hline \multirow{4}{*}{$4 \mathrm{~g}$} & 1000 & 18 & 10 & 10 & 21 & 13 & 14 & 17 \\
\hline & 500 & 16 & 8 & 7 & 17 & 9 & 10 & 11 \\
\hline & 250 & 12 & - & - & 10 & - & 7 & 8 \\
\hline & 125 & 8 & - & - & 7 & - & - & - \\
\hline \multirow{4}{*}{$4 \mathrm{~h}$} & 1000 & 12 & 18 & - & 19 & 10 & 9 & 18 \\
\hline & 500 & 10 & 12 & - & 16 & 7 & 6 & 16 \\
\hline & 250 & 9 & 9 & - & 14 & - & - & 11 \\
\hline & 125 & 9 & 6 & - & 9 & - & - & 8 \\
\hline
\end{tabular}




\begin{tabular}{|c|c|c|c|c|c|c|c|c|}
\hline \multirow{4}{*}{$4 \mathrm{i}$} & 1000 & 20 & 18 & 23 & 20 & 16 & 14 & 10 \\
\hline & 500 & 16 & 15 & 16 & 15 & 14 & 11 & 7 \\
\hline & 250 & 12 & 11 & 12 & 13 & 11 & 8 & - \\
\hline & 125 & 7 & - & 9 & 10 & 9 & 6 & - \\
\hline \multirow{4}{*}{$4 j$} & 1000 & 12 & 24 & 24 & 20 & 22 & 12 & 20 \\
\hline & 500 & 10 & 20 & 21 & 18 & 16 & 8 & 17 \\
\hline & 250 & 7 & 10 & 17 & 11 & 12 & - & 13 \\
\hline & 125 & - & - & 12 & 9 & 8 & - & 11 \\
\hline \multirow{4}{*}{$4 \mathrm{k}$} & 1000 & 15 & 12 & 9 & 18 & 17 & 24 & 18 \\
\hline & 500 & 10 & 8 & - & 15 & 13 & 20 & 14 \\
\hline & 250 & 8 & - & - & 10 & 10 & 17 & 10 \\
\hline & 125 & - & - & - & 5 & 7 & 14 & 8 \\
\hline \multirow{4}{*}{41} & 1000 & 21 & 19 & 18 & 18 & 22 & 22 & 20 \\
\hline & 500 & 18 & 16 & 15 & 14 & 18 & 19 & 17 \\
\hline & 250 & 15 & 11 & 9 & 11 & 15 & 17 & 15 \\
\hline & 125 & 10 & 7 & 7 & 9 & 10 & 13 & 10 \\
\hline \multirow{4}{*}{$4 m$} & 1000 & 20 & 14 & 12 & 24 & 24 & 20 & 16 \\
\hline & 500 & 17 & 12 & 8 & 19 & 20 & 18 & 14 \\
\hline & 250 & 12 & 8 & - & 13 & 17 & 14 & 11 \\
\hline & 125 & 8 & - & - & 11 & 12 & 10 & 7 \\
\hline \multirow{4}{*}{$4 n$} & 1000 & 16 & 14 & 10 & 22 & 19 & 15 & 20 \\
\hline & 500 & 14 & 9 & 6 & 17 & 15 & 11 & 17 \\
\hline & 250 & 8 & - & - & 13 & 10 & 7 & 11 \\
\hline & 125 & 7 & - & - & 8 & 7 & - & 8 \\
\hline \multirow{4}{*}{40} & 1000 & 20 & 20 & 12 & 14 & 17 & 18 & 19 \\
\hline & 500 & 15 & 15 & 6 & 11 & 14 & 16 & 15 \\
\hline & 250 & 10 & - & - & - & 10 & 12 & 13 \\
\hline & 125 & 15 & - & - & - & - & 9 & 10 \\
\hline \multirow{4}{*}{$4 p$} & 1000 & 20 & 12 & 14 & 12 & 21 & 13 & 10 \\
\hline & 500 & 15 & - & 11 & 10 & 15 & 11 & 8 \\
\hline & 250 & 10 & - & - & - & 13 & 10 & - \\
\hline & 125 & 7 & - & - & - & 7 & 7 & - \\
\hline \multirow{4}{*}{$4 q$} & 1000 & 20 & 20 & 19 & 17 & 19 & 20 & 21 \\
\hline & 500 & 15 & 17 & 16 & 12 & 10 & 16 & 18 \\
\hline & 250 & - & 12 & 11 & 9 & 7 & 14 & 16 \\
\hline & 125 & - & 9 & 6 & 7 & - & 9 & 12 \\
\hline
\end{tabular}




\begin{tabular}{|c|c|c|c|c|c|c|c|c|}
\hline \multirow{4}{*}{$4 \mathrm{r}$} & 1000 & 25 & 21 & 20 & 12 & 22 & 22 & 23 \\
\hline & 500 & 18 & 19 & 17 & 9 & 19 & 19 & 20 \\
\hline & 250 & 15 & 14 & 11 & - & 15 & 15 & 19 \\
\hline & 125 & 12 & 10 & 8 & - & 11 & 11 & 14 \\
\hline \multirow{4}{*}{$4 s$} & 1000 & 22 & 21 & 12 & 22 & 16 & 18 & 20 \\
\hline & 500 & 17 & 15 & 9 & 17 & 12 & 13 & 17 \\
\hline & 250 & 12 & 13 & - & 11 & 8 & 9 & 13 \\
\hline & 125 & 8 & 9 & - & 9 & - & 7 & 11 \\
\hline \multirow{4}{*}{$4 t$} & 1000 & 12 & 14 & - & 17 & 7 & 14 & - \\
\hline & 500 & 11 & 10 & - & 15 & - & 11 & - \\
\hline & 250 & 8 & 8 & - & 11 & - & 8 & - \\
\hline & 125 & - & - & - & 7 & - & - & - \\
\hline \multirow{4}{*}{$4 u$} & 1000 & 24 & 22 & 22 & 10 & 21 & 20 & 22 \\
\hline & 500 & 20 & 22 & 18 & 8 & 18 & 16 & 18 \\
\hline & 250 & 16 & 18 & 14 & - & 12 & 13 & 15 \\
\hline & 125 & 10 & 14 & 10 & - & 9 & 9 & 9 \\
\hline \multirow{4}{*}{$4 v$} & 1000 & 18 & 19 & 17 & 14 & 24 & 17 & 19 \\
\hline & 500 & 16 & 13 & 13 & 12 & 19 & 15 & 15 \\
\hline & 250 & 15 & 10 & 10 & 7 & 15 & 10 & 13 \\
\hline & 125 & 12 & - & 8 & - & 12 & 7 & 11 \\
\hline \multirow{4}{*}{$4 w$} & 1000 & 15 & 8 & - & 18 & 13 & 20 & - \\
\hline & 500 & 14 & - & - & 14 & 9 & 17 & - \\
\hline & 250 & 8 & - & - & 11 & 7 & 15 & - \\
\hline & 125 & 8 & - & - & 8 & - & 12 & - \\
\hline $\mathrm{AMX}^{\mathrm{b}}$ & $10 \mu \mathrm{g} / \mathrm{disc}$ & 11 & 10 & 19 & 12 & 20 & 18 & 10 \\
\hline $\mathrm{AK}^{\mathrm{b}}$ & $10 \mu \mathrm{g} / \mathrm{disc}$ & 18 & 17 & 11 & 19 & 20 & 18 & 10 \\
\hline $\mathrm{CTR}^{\mathrm{b}}$ & $10 \mu \mathrm{g} / \mathrm{disc}$ & 8 & 9 & 16 & 13 & 17 & 16 & 9 \\
\hline $\mathrm{CIP}^{\mathrm{b}}$ & $30 \mu \mathrm{g} / \mathrm{disc}$ & 13 & 11 & 17 & 22 & 21 & 23 & 12 \\
\hline $\mathrm{DMSO}^{\mathrm{c}}$ & & 5 & 5 & 0 & 5 & 6 & 0 & 6 \\
\hline
\end{tabular}

Abbreviations: ${ }^{(a)}$ Microorganisms, E.c.: Escherichia Coli; P.a.: Pseudomonas Aeruginosa; V.s.: Viridans Streptococci; C.s.: Coagulase negative Staphylococcus; P.s.: Proteus Species; S.s.: Salmonella Species; K.s.: Klebsiella Species; ${ }^{(b)}$ Antibiotics, AMX: Amoxycillin; AK: Amikacin; CTR: Ceftriazone; CIP: Ciprofloxacin.

${ }^{c}$ DMSO used as the control.

${ }^{\mathrm{d}}$ The data represents the mean values of two replicates

6-10 mm, low activity; 11-15 mm, moderate activity; 16-19 mm, high activity; more than $20 \mathrm{~mm}$, very high activity. 


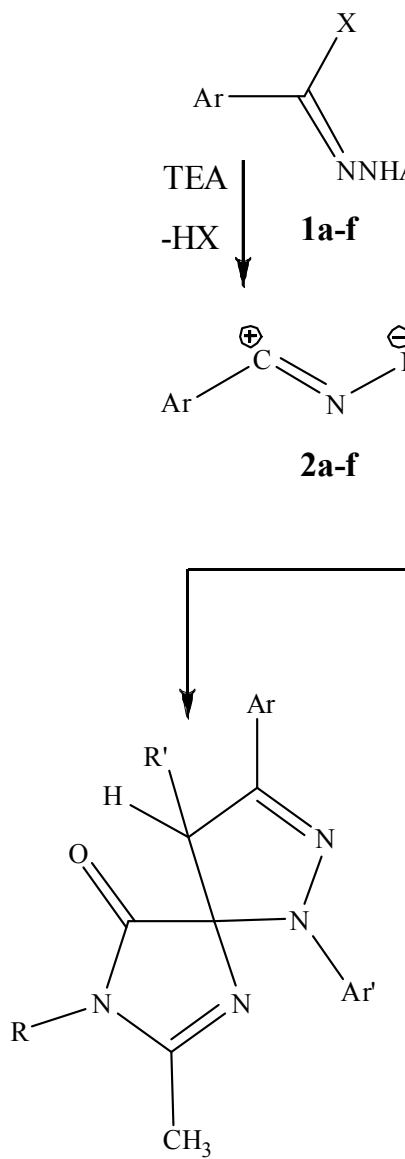

4a-w

$$
\text { Ar / Ar' / X }
$$

1a, 2a, $\mathrm{Ph} / \mathrm{Ph} / \mathrm{Cl}$

1b, 2b, 4- $\mathrm{ClC}_{6} \mathrm{H}_{4} / \mathrm{Ph} / \mathrm{Cl}$

1c, 2c, 2,4- $\mathrm{Cl}_{2} \mathrm{C}_{6} \mathrm{H}_{3} / 4-\mathrm{NO}_{2} \mathrm{C}_{6} \mathrm{H}_{4} / \mathrm{Br}$

1d, 2d, 4- $\mathrm{FC}_{6} \mathrm{H}_{4} / 4-\mathrm{NO}_{2} \mathrm{C}_{6} \mathrm{H}_{4} / \mathrm{Br}$

1e, $2 \mathbf{e}, \mathrm{Ph} / 4-\mathrm{NO}_{2} \mathrm{C}_{6} \mathrm{H}_{4} / \mathrm{Br}$

1f, 2f, 2-furyl / 4- $\mathrm{NO}_{2} \mathrm{C}_{6} \mathrm{H}_{4} / \mathrm{Cl}$

$$
\mathrm{R} / \mathrm{R}^{\prime} / \mathrm{Ar} / \mathrm{Ar}^{\prime}
$$

4a, $\mathrm{Ph} / \mathrm{Ph} / \mathrm{Ph} / \mathrm{Ph}$

4b, $\mathrm{Ph} / \mathrm{Ph} / 4-\mathrm{ClC}_{6} \mathrm{H}_{4} / \mathrm{Ph}$

4c, $\mathrm{Ph} / \mathrm{Ph} / 2,4-\mathrm{Cl}_{2} \mathrm{C}_{6} \mathrm{H}_{3} / 4-\mathrm{NO}_{2} \mathrm{C}_{6} \mathrm{H}_{4}$

4d, $\mathrm{Ph} / \mathrm{Ph} / 2$-furyl / 4- $\mathrm{NO}_{2} \mathrm{C}_{6} \mathrm{H}_{4}$

4e, $\mathrm{Ph} / 4-\mathrm{CH}_{3} \mathrm{C}_{6} \mathrm{H}_{4} / \mathrm{Ph} / \mathrm{Ph}$

4f, $\mathrm{Ph} / 4-\mathrm{CH}_{3} \mathrm{C}_{6} \mathrm{H}_{4} / 4-\mathrm{ClC}_{6} \mathrm{H}_{4} / \mathrm{Ph}$

4g, $\mathrm{Ph} / 4-\mathrm{CH}_{3} \mathrm{C}_{6} \mathrm{H}_{4} / 4-\mathrm{FC}_{6} \mathrm{H}_{4} / 4-\mathrm{NO}_{2} \mathrm{C}_{6} \mathrm{H}_{4}$

4h, $\mathrm{Ph} / 4-\mathrm{CH}_{3} \mathrm{C}_{6} \mathrm{H}_{4} / \mathrm{Ph} / 4-\mathrm{NO}_{2} \mathrm{C}_{6} \mathrm{H}_{4}$

4i, $\mathrm{Ph} / 4-\mathrm{CH}_{3} \mathrm{C}_{6} \mathrm{H}_{4} / 2$-furyl / 4- $\mathrm{NO}_{2} \mathrm{C}_{6} \mathrm{H}_{4}$

4j, $\mathrm{Ph} / 2-\mathrm{CH}_{3} \mathrm{OC}_{6} \mathrm{H}_{4} / \mathrm{Ph} / \mathrm{Ph}$

4k, Ph / 2- $\mathrm{CH}_{3} \mathrm{OC}_{6} \mathrm{H}_{4} / 4-\mathrm{ClC}_{6} \mathrm{H}_{4} / \mathrm{Ph}$<smiles>[R4]C=C1N=C(C)N([R])C1=O</smiles>

3a-f

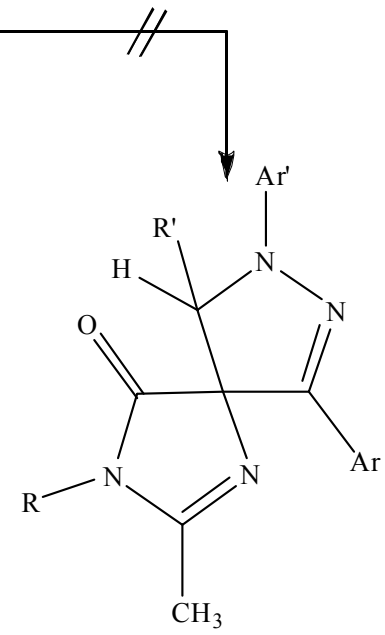

5
$\mathrm{R} / \mathrm{R}^{\prime}$

3a, $\mathrm{Ph} / \mathrm{Ph}$

3b, $\mathrm{Ph} / 4-\mathrm{CH}_{3} \mathrm{C}_{6} \mathrm{H}_{4}$

3c, $\mathrm{Ph} / 2-\mathrm{CH}_{3} \mathrm{OC}_{6} \mathrm{H}_{4}$

3d, $\mathrm{Ph} / 3-\mathrm{CH}_{3} \mathrm{OC}_{6} \mathrm{H}_{4}$

3e, $\mathrm{Ph} / 4-\mathrm{C}_{2} \mathrm{H}_{5} \mathrm{OC}_{6} \mathrm{H}_{4}$

3f, $4-\mathrm{CH}_{3} \mathrm{OC}_{6} \mathrm{H}_{4} / 2-\mathrm{CH}_{3} \mathrm{OC}_{6} \mathrm{H}_{4}$

$$
\mathrm{R} / \mathrm{R} \text { / / } \mathrm{Ar} / \mathrm{Ar}^{\prime}
$$

4l, $\mathrm{Ph} / 2-\mathrm{CH}_{3} \mathrm{OC}_{6} \mathrm{H}_{4} / 2,4-\mathrm{Cl}_{2} \mathrm{C}_{6} \mathrm{H}_{3} / 4-\mathrm{NO}_{2} \mathrm{C}_{6} \mathrm{H}_{4}$ $4 \mathbf{m}, \mathrm{Ph} / 2-\mathrm{CH}_{3} \mathrm{OC}_{6} \mathrm{H}_{4} / 2$-furyl / 4- $\mathrm{NO}_{2} \mathrm{C}_{6} \mathrm{H}_{4}$

4n, $\mathrm{Ph} / 3-\mathrm{CH}_{3} \mathrm{OC}_{6} \mathrm{H}_{4} / \mathrm{Ph} / \mathrm{Ph}$

4o, $\mathrm{Ph} / 3-\mathrm{CH}_{3} \mathrm{OC}_{6} \mathrm{H}_{4} / 4-\mathrm{ClC}_{6} \mathrm{H}_{4} / \mathrm{Ph}$

4p, $\mathrm{Ph} / 3-\mathrm{CH}_{3} \mathrm{OC}_{6} \mathrm{H}_{4} / 4-\mathrm{FC}_{6} \mathrm{H}_{4} / 4-\mathrm{NO}_{2} \mathrm{C}_{6} \mathrm{H}_{4}$

4q, $\mathrm{Ph} / 4-\mathrm{C}_{2} \mathrm{H}_{5} \mathrm{OC}_{6} \mathrm{H}_{4} / \mathrm{Ph} / \mathrm{Ph}$

4r, $\mathrm{Ph} / 4-\mathrm{C}_{2} \mathrm{H}_{5} \mathrm{OC}_{6} \mathrm{H}_{4} / 4-\mathrm{ClC}_{6} \mathrm{H}_{4} / \mathrm{Ph}$

4s, $\mathrm{Ph} / 4-\mathrm{C}_{2} \mathrm{H}_{5} \mathrm{OC}_{6} \mathrm{H}_{4} / 2,4-\mathrm{Cl}_{2} \mathrm{C}_{6} \mathrm{H}_{3} / 4-\mathrm{NO}_{2} \mathrm{C}_{6} \mathrm{H}_{4}$

4t, $\mathrm{Ph} / 4-\mathrm{C}_{2} \mathrm{H}_{5} \mathrm{OC}_{6} \mathrm{H}_{4} / 2$-furyl / 4- $\mathrm{NO}_{2} \mathrm{C}_{6} \mathrm{H}_{4}$

$4 \mathbf{u}, 4-\mathrm{CH}_{3} \mathrm{OC}_{6} \mathrm{H}_{4} / 2-\mathrm{CH}_{3} \mathrm{OC}_{6} \mathrm{H}_{4} / \mathrm{Ph} / \mathrm{Ph}$

$4 \mathbf{v}, 4-\mathrm{CH}_{3} \mathrm{OC}_{6} \mathrm{H}_{4} / 2-\mathrm{CH}_{3} \mathrm{OC}_{6} \mathrm{H}_{4} / 4-\mathrm{ClC}_{6} \mathrm{H}_{4} / \mathrm{Ph}$

Scheme 1 